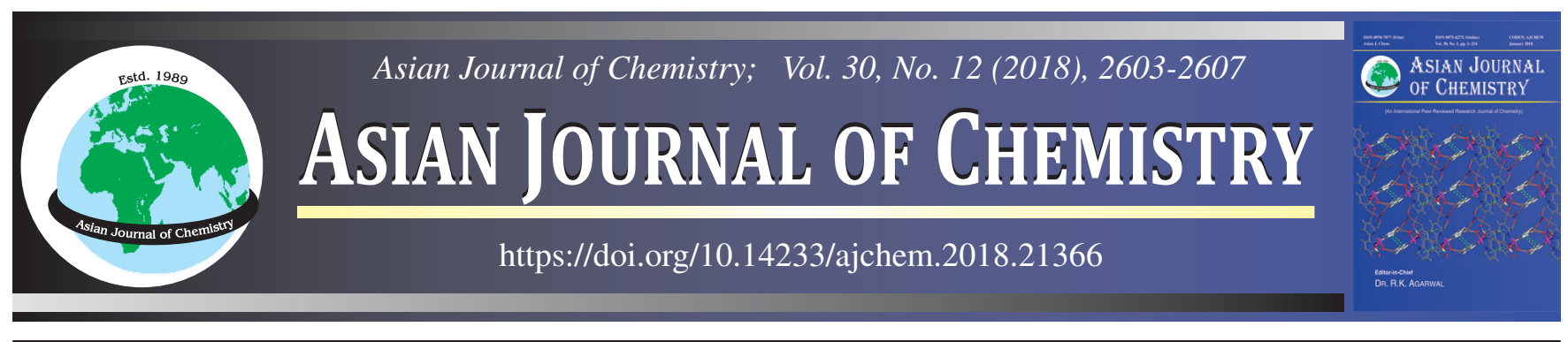

\title{
Synthesis and Preliminary Anticancer Activity Study of New 6-Mercaptopurine Derivatives
}

Dunya L. Al-Duhaidahawi

Department of Pharmaceutical Chemistry, College of Pharmacy, Kufa University, Al-Najaf, Iraq

Corresponding author: E-mail: dunyal.mohammed@uokufa.edu.iq

Received: 6 April 2018;

Accepted: 24 September 2018;

Published online: 31 October 2018;

AJC-19118

The synthesis of asymmetrical disulfides is an essential alteration in progressive investigation in pharmaceutical chemistry. The current research pointed at the design and synthesis of novel antitumor products from 6-mercaptopurine by the introduction of heterocyclic substituted 1,2,4 triazole-sulfhydryl moiety as a bioisostere at SH group. To this functional group, a series of $12 s$-triazole derivatives with different alkyl and aralkyl substituents as functionalized side chains of disulfide derivative were synthesized utilizing 1-chloro-benzotriazole as oxidizing agents. Structure of compounds was characterized by elemental microanalysis and spectral analysis followed by in vitro cytotoxic activities against CLL-119, L1210 and HL60 cell lines was assessed by MTT test method. The results from the primary test showed that the introduction of substituted 1,2,4-triazole-sulfhydryl highly improve the therapeutic efficacy of drug. Compounds $\mathbf{3}, \mathbf{5}$ and 8 show best anti-CCL-119 activity.

Keywords: 6-Mercaptopurine, Anticancer, Disulfide, 1,2,4-Triazole, MTT.

\section{INTRODUCTION}

Mercaptopurine (Puri-Nethol ${ }^{\mathrm{TM}}$; 6-MP) is used almost exclusively as sustaining therapy for acute leukemia $[1,2]$. The free-base structure is transformed by sensitive cancer cells toward the ribonucleotide 6-mercaptopurin-9-yl (MPRP), that emerges from the interaction of the drug with 5-phosphoribosyl transferase $[3,4]$. For the conventional purine anticancer drug, 6-mercaptopurine central pathways of deactivation (Fig. 1) include methylation of SH group by thiopurine-S-methyltransferase (TPMT) and oxidation by the enzyme xanthine oxidase (XO) [5]. Heterocyclic analogs of 6-mercaptopurine, such as azathioprine, were invented to shield it from metabolic effects $[6,7]$. In spite of azathioprine holds antitumor activity, it isn't fundamentally superior to 6-mercaptopurine [8]. It has an essential part in organ transplants as an immuno-suppressive agent [9]. Today, these thiopurine bases continue to be basic means in the inception and maintenance therapy in patients with myelocytic and intense lymphocytic leukemia $[10,11]$. Regardless of its set up clinical significance, 6-mercaptopurine has particular restorative drawbacks [12], which have kept on stimulating the search of purine analogs enhancing therapeutic adequacy.<smiles>S=c1[nH]cnc2nc[nH]c12</smiles>

Fig 1. 6-Mercaptopurine structure along with tautomer forms

Significant attempts performed to develop distinct novel mercaptopurine analogs and their nucleosides to enhance the antitumor effectiveness by coupling sulfhydryl with different heterocyclic rings as disulfide prodrugs as enzyme thiopurine S-methyltransferase (TPMT) is engaged, partly, for the deactivation of 6-mercaptopurine [13,14]. Thiopurine $S$-methyltransferase (TPMT) accelerate the methylation of 6-mercaptopurine into 6-methylmercaptopurine that lack activity the added methyl group limits mercaptopurine from additional transformation into effective, cytotoxic thioguanine nucleotide (TGN) products of metabolism so masking sulfhydryl group, as disulfide will limit such deactivation [15]. There are only a few examples of analogs of drugs containing an SH group like $S$-soft alkyl analogs of 6-mercaptopurine (6-MP) [16] and $S$-allylthio-6-mercaptopurine (SA-6MP), $S$-allyl-thio6-mercaptopurine riboside (SA-6MPR) [17] which was examined for antileukemic action, exploiting a humanoid-

This is an open access journal, and articles are distributed under the terms of the Creative Commons Attribution-NonCommercial 4.0 International (CC BY-NC 4.0) License, which allows others to copy and redistribute the material in any medium or format, remix, transform, and build upon the material, as long as appropriate credit is given and the new creations are licensed under the identical terms. 
mouse B-CLL type. 1,2,4-Triazole is a moiety integrated in to abroad diversity of compounds that shown antitumor activity, triazole moiety is examined widely in nucleoside analogs anticancer medications, kinase blockers, tubulin polymerization inhibitors, aromatase and sulfatase blockers as well as metal complex compounds as anticancer [18-20]. Lin et al. [21] reported a sequence of 1-acyl-1H-[1,2,4]triazole-3,5-diamine derivatives and discovered them to be cyclin-dependent kinase (CDK) blockers. Zhang et al. [22] produced 1,2,4-triazole compounds containing pyridine as a promising adhesion kinase (FAK) blockers and antitumor agents. Significant attempts have been performed in this study to make another novel mercaptopurine analogs to improve the antitumor efficacy by sulfhydryl with different heterocyclic groups bearing 1,2,4 triazole sulfahydryl as disulfide prodrugs such chimeric molecules would be a rational design to improve affinity and efficacy compared to the parent drugs.

\section{EXPERIMENTAL}

Compounds and reagents obtained from Fluka and BDH chemical company were of reasonable grade type used without extra refinement. The IR bands were taken on a Nicolet 6700 FT-IR spectrophotometer (Thermo Nicolet Corp., Madison, WI, USA), and the spectra recorded in $\mathrm{cm}^{-1}$. Nuclear magnetic resonance (NMR) bands reported by an AVANCE III $500 \mathrm{MHz}$ spectrometer (Bruker, Billerica, MA, USA), DMSO utilized as a solvent and the data imported in $\delta$ ppm frequency of resonance are shielded from tetramethylsilane the reference compound.
Elemental microanalysis was made on an Elementary Vario El III Carlo Erba 1108 elemental analyzer Carlo Erba Reagent SpA, Rodano, Italy.

Statistical analysis: The results were expressed as the mean \pm standard deviation and the arithmetical outcome of fluctuations was closed applying the one-path measure of difference (ANOVA) by the SPSS 17.0 arithmetical programming bundle. Varieties were reflected significant at $p<0.05$. The information shows up as mean $\pm \operatorname{SD}(n=3)$.

Synthesis: A unique construction of asymmetrical disulfides is reported. Thiol compound (R1SH) reaction with 1-chlorobenzotriazole $(\mathrm{BtCl})$ at $-(20-28)^{\circ} \mathrm{C}$ in dichloromethane some time use DMF according to the solubility of reactants yields a high-productive regeneration to R1SBt without significant production of the symmetrical disulfide R1SSR1. R1SBt is later reacted with $\mathrm{R}^{2} \mathrm{SH}$ to make the asymmetrical disulfide in $60-85 \%$ yield in a one synthetic path with green quality that by passes the application of poisonous and tough rusting elements (Scheme-I). The development of reaction checked by LC/MS and yield mostly refined by column chromatography utilizing light petroleum/EtOAc combinations (1:4).

Synthesis of 5-((7H-purin-6-yl)disulfanyl)- $N$,4-diphenyl4H-1,2,4-triazol-3-amine (compound 1): A stirred mix of 1-chorobenzotriazole $(0.61 \mathrm{~g}, 4 \mathrm{mmol})$ and benzotriazole $(0.32 \mathrm{~g}$, $20.7 \mathrm{mmol})$ in $\mathrm{CH}_{2} \mathrm{Cl}_{2}(30 \mathrm{~mL})$ underneath $\mathrm{N}_{2}$ at $-20{ }^{\circ} \mathrm{C}$ was supplemented dropwise a suspension of 4-phenyl-5-(phenylamino)-4H-1,2,4-triazole-3-thiol as $\mathrm{R}^{1} \mathrm{SH}(0.5 \mathrm{~g}, 2.7 \mathrm{mmol})$ in $\mathrm{CH}_{2} \mathrm{Cl}_{2}(5 \mathrm{~mL})$. The mixture was permitted to stir for $2 \mathrm{~h}$<smiles>Cln1nnc2ccccc21</smiles>

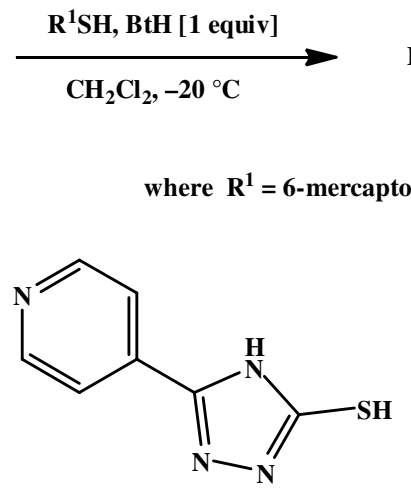

2<smiles>Oc1ccccc1-c1nnc(S)[nH]1</smiles>

5<smiles>Cc1nnc(S)s1</smiles><smiles>CCCCCn1c(O)nnc1S</smiles>

6<smiles>Sc1nc[nH]n1</smiles>

10
$\mathbf{R}^{1}$ SBt

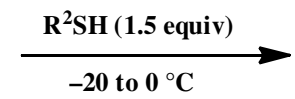<smiles>Nn1c(S)nnc1-c1ccccc1</smiles><smiles>Cn1cnnc1S</smiles>

7<smiles>Sc1nnc(-c2ccco2)[nH]1</smiles>

11<smiles>Nn1c(S)nnc1-c1ccccn1</smiles><smiles>Cn1c(O)nnc1S</smiles>

Scheme-I: Synthesis of target compounds 
with regular heating to $-10^{\circ} \mathrm{C}$. Later, 6-mercaptopurine as $\mathrm{R}^{2} \mathrm{SH}(0.6 \mathrm{~g}$, four $\mathrm{mmol})$ in $\mathrm{CH}_{2} \mathrm{Cl}_{2} / \mathrm{DMF}(6 \mathrm{~mL})$ was then sequentially adjoined at $-20^{\circ} \mathrm{C}$ and the mixture stirred at $0{ }^{\circ} \mathrm{C}$ for $0.5 \mathrm{~h}$. The synthetic step was then terminated with a liquid of $\mathrm{Na}_{2} \mathrm{~S}_{2} \mathrm{O}_{3}(0.50 \mathrm{~g}$ in $10 \mathrm{~mL}$ water $)$ simultaneously with drenched aqueous $\mathrm{NaHCO}_{3}(20 \mathrm{~mL})$, with prompt agitating at $0{ }^{\circ} \mathrm{C}$ for 20 min prior implying liquid extraction with $\mathrm{CH}_{2} \mathrm{Cl}_{2}(3 \times$ $100 \mathrm{~mL}$ ). The collected organic extracts were evaporated over anhydrous $\mathrm{MgSO}_{4}$, filtrated and concentrated under diminished pressure. The raw material refined by column chromatographic technique employing light petroleum/EtOAc combinations to furnish disulphide with $89 \%$ yield as dark beige solid. m.p. $265^{\circ} \mathrm{C} .{ }^{1} \mathrm{H}$ NMR (DMSO- $\left.d_{6}\right) \delta 14.76$ (s, 1H, NH 6MP), 9.39 (s, 1H, NH linked triazole), 8.95 (s, 1H), 8.12 (s, $1 \mathrm{H}), 7.71(\mathrm{~d}, J=6.8 \mathrm{~Hz}, 2 \mathrm{H}), 7.60(\mathrm{dd}, J=16.8,7.6 \mathrm{~Hz}, 4 \mathrm{H})$, $7.45(\mathrm{t}, J=7.4 \mathrm{~Hz}, 2 \mathrm{H}), 7.30(\mathrm{t}, J=7.4 \mathrm{~Hz}, 2 \mathrm{H}), 7.02(\mathrm{t}, J=$ $7.5 \mathrm{~Hz}, 1 \mathrm{H})$. IR $\left(\mathrm{KBr}, v_{\max }, \mathrm{cm}^{-1}\right): 1164(\mathrm{C}-\mathrm{S}), 1586(\mathrm{C}=\mathrm{N})$, $1429(\mathrm{C}=\mathrm{C}), 1363(\mathrm{C}=\mathrm{C}), 1123(\mathrm{C}=\mathrm{C}), 727(\mathrm{C}-\mathrm{S}), 542(\mathrm{~S}-\mathrm{S})$. CHNS analysis calcd. (found) \%: C, 54.53 (54.64); H, 3.37 (3.51); N, 26.78 (26.62); S, 15.32 (15.59). HRMS: $m / z\left(\mathrm{EI}^{+}\right)$ calcd. for $\mathrm{C}_{19} \mathrm{H}_{14} \mathrm{~N}_{8} \mathrm{~S}_{2}\left(\mathrm{M}+\mathrm{H}^{+}\right) 418.0783$, found $419.0994\left(\mathrm{M}+\mathrm{H}^{+}\right)$.

6-((5-(Pyridin-4-yl)-4H-1,2,4-triazol-3-yl)disulfanyl)7H-purine (compound 2): Dark-yellow solid, Yield: $79 \%$, m.p. $205{ }^{\circ} \mathrm{C} .{ }^{1} \mathrm{H}$ NMR (DMSO-d $) \delta 14.79$ (s, 1H, NH-6MP), 9.273 (s, 1H, NH linked triazole), $8.72(\mathrm{~d}, J=5.1 \mathrm{~Hz}, 2 \mathrm{H}, \mathrm{CH}=\mathrm{N}$ Pyr), 8.12 (s, 1H, NH-CH=N 6-MP), 7.92 (d, J = 5.2 Hz, 2H, $\mathrm{CH}=\mathrm{C}$ pyr). IR $\left(\mathrm{KBr}, \nu_{\max }, \mathrm{cm}^{-1}\right): 1164(\mathrm{C}-\mathrm{S}), 1586(\mathrm{C}=\mathrm{N}), 1429$ $(\mathrm{C}=\mathrm{C}), 1363(\mathrm{C}=\mathrm{C}), 1123(\mathrm{C}=\mathrm{C}),(\mathrm{C}-\mathrm{S})$ 730, 542(S-S). CHNS analysis calcd. (found) \%: C, 43.89 (44.02), H, 2.46 (2.51), $\mathrm{N}, 34.12$ (34.52) S, 19.53 (19.59). HRMS: $m / z\left(\mathrm{EI}^{+}\right)$calcd. for $\mathrm{C}_{12} \mathrm{H}_{8} \mathrm{~N}_{8} \mathrm{~S}_{2}\left(\mathrm{M}+\mathrm{H}^{+}\right)$328.0313, found $329.0976\left(\mathrm{M}+\mathrm{H}^{+}\right)$.

3-((7H-Purin-6-yl)disulfanyl)-5-phenyl-4H-1,2,4triazol-4-amine (compound 3): Off-white solid, Yield: $73 \%$, m.p. $287{ }^{\circ} \mathrm{C},{ }^{1} \mathrm{H}$ NMR (DMSO- $\left.d_{6}\right) \delta 14.802$ (s, $\left.1 \mathrm{H}, \mathrm{NH} 6-\mathrm{MP}\right)$, 9.003 (s, 1H, CH=N 6-MP), 8.224 (s, 1H, CH arom.), 8.0917.984 (m, 2H), 7.503 (d, $J=5.5 \mathrm{~Hz}, 3 \mathrm{H}, \mathrm{CH}=\mathrm{CH}-\mathrm{CH}$ arom.), $4.956\left(\mathrm{~s}, 2 \mathrm{H}, \mathrm{NH}_{2}\right)$. IR (KBr, $\left.v_{\max }, \mathrm{cm}^{-1}\right): 1164(\mathrm{C}-\mathrm{S}), 1586$ $(\mathrm{C}=\mathrm{N}), 1167(\mathrm{C}-\mathrm{OH}), 1429(\mathrm{C}=\mathrm{C}), 1363(\mathrm{C}=\mathrm{C}), 1123(\mathrm{C}=\mathrm{C})$, $727(\mathrm{C}-\mathrm{S}), 542(\mathrm{~S}-\mathrm{S})$. CHNS analysis calcd. (found) \%: C, 45.60 (45.87), H, 2.94 (2.67), N, 32.73 (32.31), S, 18.73 (18.49). HRMS: $m / z\left(\mathrm{EI}^{+}\right)$calcd. for $\mathrm{C}_{13} \mathrm{H}_{10} \mathrm{~N}_{8} \mathrm{~S}_{2} 342.0470\left(\mathrm{M}+\mathrm{H}^{+}\right)$, found $343.0442\left(\mathrm{M}+\mathrm{H}^{+}\right)$.

3-((7H-Purin-6-yl)disulfanyl)-5-(pyridin-2-yl)-4H-1,2,4triazol-4-amine (compound 4): Yellowish powder, Yield: $80 \%$, m.p. $175^{\circ} \mathrm{C} .{ }^{1} \mathrm{H}$ NMR (DMSO- $d_{6}$ ): $\delta 15.35$ (s, $1 \mathrm{H}, \mathrm{NH}$ 6-MP), 9.27 (s, 1H, CH=N 6-MP), 8.69 (d, J=5.0 Hz, 1H, CH=C pyr.), 8.12 (s, 1H, NH-CH=N 6-MP), 7.94 (d, $J=8.0 \mathrm{~Hz}, 1 \mathrm{H}, \mathrm{CH}=\mathrm{CH}$ pyr.), $7.78(\mathrm{t}, J=8.0 \mathrm{~Hz}, 1 \mathrm{H}, \mathrm{CH}=\mathrm{CH}$ pyr. $), 7.40$ (t, $J=6.6 \mathrm{~Hz}, 1 \mathrm{H}$, $\mathrm{CH}=\mathrm{CH}$ pyr.), 5.39 (s, 2H, $\left.\mathrm{NH}_{2}\right)$. IR ( $\left.\mathrm{KBr}, v_{\max }, \mathrm{cm}^{-1}\right): 1167$ $(\mathrm{C}-\mathrm{S}), 1588(\mathrm{C}=\mathrm{N}), 1426(\mathrm{C}=\mathrm{C}), 1368(\mathrm{C}=\mathrm{C}), 1127(\mathrm{C}=\mathrm{C}), 728$ (C-S), $546(\mathrm{~S}-\mathrm{S})$. CHNS analysis calcd. (found) \%: C, 41.97 (41.87), H, 2.64 (2.67) N, 36.71 (36.61), S, 18.68 (18.59). HRMS: $m / z\left(\mathrm{EI}^{+}\right)$calcd. for $\mathrm{C}_{12} \mathrm{H}_{9} \mathrm{~N}_{9} \mathrm{~S}_{2} 343.0422\left(\mathrm{M}+\mathrm{H}^{+}\right)$, found 344.0446 $\left(\mathrm{M}+\mathrm{H}^{+}\right)$.

2-(5-((7H-Purin-6-yl)disulfanyl)-4H-1, 2,4-triazol-3-yl) phenol (compound 5): Beige powder, Yield: $80 \%$, m.p. 198 ${ }^{\circ} \mathrm{C},{ }^{1} \mathrm{H}$ NMR (DMSO- $d_{6}$ ): $\delta 14.81$ (s, 1H, NH 6-MP), 11.32 (s, 1H, OH), 9.11 (s, 1H, CH=N 6-MP), 8.12 (s, 1H, CH=N 6-MP),
7.49 (d, $J=7.4 \mathrm{~Hz}, 1 \mathrm{H}, \mathrm{CH}=\mathrm{CH}$ arom.), 7.27 (t, $J=7.6 \mathrm{~Hz}$, 1H, $\mathrm{CH}=\mathrm{CH}$ arom.), 7.12 (t, $J=7.6 \mathrm{~Hz}, 1 \mathrm{H}, \mathrm{CH}-\mathrm{CH}$ arom.), 7.07 (d, $J=7.2 \mathrm{~Hz}, 1 \mathrm{H}, \mathrm{CH}=\mathrm{CH}$ arom.). IR $\left(\mathrm{KBr}, v_{\max }, \mathrm{cm}^{-1}\right): 3400$ (broad OH), $1164(\mathrm{C}-\mathrm{S}), 1584(\mathrm{C}=\mathrm{N}), 1167(\mathrm{C}-\mathrm{OH}), 1426(\mathrm{C}=\mathrm{C})$, $1365(\mathrm{C}=\mathrm{C}), 1128(\mathrm{C}=\mathrm{C}), 720(\mathrm{C}-\mathrm{S}), 530(\mathrm{~S}-\mathrm{S})$. CHNS analysis calcd. (found) \%: C, 45.47 (45.57), H, 2.64 (2.66), N, 28.55 (28.61) $\mathrm{S}, 18.68$ (18.59). HRMS: $m / z\left(\mathrm{EI}^{+}\right)$calcd. for $\mathrm{C}_{13} \mathrm{H}_{9} \mathrm{~N}_{7} \mathrm{OS}_{2} 343.031$ $\left(\mathrm{M}+\mathrm{H}^{+}\right)$, found $343.046\left(\mathrm{M}+\mathrm{H}^{+}\right)$.

5-((7H-purin-6-yl)disulfanyl)-4-pentyl-4H-1,2,4-triazol -3-ol (compound 6): Yellowish powder, Yield: $77 \%$, m.p. 225 ${ }^{\circ} \mathrm{C} .{ }^{1} \mathrm{H}$ NMR (DMSO- $d_{6}$ ): $\delta 14.32$ (s, 1H, NH 6-MP), 11.34 (s, 1H, OH), 8.98 (s, 1H, CH=N 6-MP), 8.12 (s, 1H, CH-NH 6MP), 4.00 (t, $J=7.6 \mathrm{~Hz}, 2 \mathrm{H}, \mathrm{CH}_{2}-\mathrm{N}$ alkyl), 1.85 (p, $J=7.7$ $\mathrm{Hz}, 2 \mathrm{H}, \mathrm{CH}_{2}-\mathrm{CH}_{2}-\mathrm{N}$ ), 1.28 (q, J = 8.3 Hz, 4H, $\mathrm{CH}_{2}-\mathrm{CH}_{2}$ ), 0.92$0.84\left(\mathrm{~m}, 3 \mathrm{H}, \mathrm{CH}_{3}\right)$. IR $\left(\mathrm{KBr}, \mathrm{v}_{\max }, \mathrm{cm}^{-1}\right): 1164(\mathrm{C}-\mathrm{S}), 1584(\mathrm{C}=\mathrm{N})$, $1166(\mathrm{C}-\mathrm{OH}), 1426(\mathrm{C}=\mathrm{C}), 1365(\mathrm{C}=\mathrm{C}), 1123(\mathrm{C}=\mathrm{C}), 713(\mathrm{C}-\mathrm{S})$, 535 (S-S). CHNS analysis calcd. (found) \%: C, 42.71 (42.67), H, 4.48 (4.56), N, 29.06 (29.11), S, 19.01 (19.09). HRMS: $m / z$ $\left(\mathrm{EI}^{+}\right.$) calcd. for $\mathrm{C}_{12} \mathrm{H}_{15} \mathrm{~N}_{7} \mathrm{OS}_{2} 337.0779\left(\mathrm{M}+\mathrm{H}^{+}\right)$, found 338.069 $\left(\mathrm{M}+\mathrm{H}^{+}\right)$.

6-((4-Methyl-4H-1,2,4-triazol-3-yl)disulfanyl)-7H-purine (compound 7): Dark yellow solid, Yield: $85 \%$, m.p. $252{ }^{\circ} \mathrm{C}$. ${ }^{1} \mathrm{H}$ NMR (DMSO- $d_{6}$ ): $\delta 15.38$ (s, 1H, NH 6-MP), 8.98 (d, $J=$ $8.9 \mathrm{~Hz}, 2 \mathrm{H}, \mathrm{CH}=\mathrm{N}$ triazole), 8.12 (s, 1H, CH=N 6-MP), 3.67 (s, 3H, $\left.\mathrm{CH}_{3}\right)$. IR $\left(\mathrm{KBr}, v_{\max }, \mathrm{cm}^{-1}\right): 2850\left(\mathrm{C}-\mathrm{H}\right.$ str. $\left.\mathrm{CH}_{3}\right), 1169(\mathrm{C}-\mathrm{S})$, 1587 $(\mathrm{C}=\mathrm{N}), 1428(\mathrm{C}=\mathrm{C}), 1364(\mathrm{C}=\mathrm{C}), 1120(\mathrm{C}=\mathrm{C}), 713(\mathrm{C}-\mathrm{S})$, $565(\mathrm{~S}-\mathrm{S})$. CHNS analysis calcd. (found) $\%: 36.22(36.42) ; \mathrm{H}$, 2.66 (2.56); N, 36.95 (36.91); S, 24.17 (24.11). HRMS: $\mathrm{m} / \mathrm{z}$ $\left(\mathrm{EI}^{+}\right)$calcd. for $\mathrm{C}_{12} \mathrm{H}_{15} \mathrm{~N}_{7} \mathrm{OS}_{2} 265.0204\left(\mathrm{M}+\mathrm{H}^{+}\right)$, found 266.0235 $\left(\mathrm{M}+\mathrm{H}^{+}\right)$.

5-((7H-Purin-6-yl)disulfanyl)-4-methyl-4H-1,2,4-triazol3-ol (compound 8): Off white powder, Yield: $83 \%$, m.p. 159 ${ }^{\circ} \mathrm{C}$. ${ }^{1} \mathrm{H}$ NMR (DMSO- $d_{6}$ ): $\delta 14.79$ (s, 1H, NH 6-MP), 11.34 (s, $1 \mathrm{H}$, $\mathrm{OH}), 8.89$ (s, 1H, CH=N 6-MP), 8.12 (s, 1H, CH=N), 3.80 (s, $\left.3 \mathrm{H}, \mathrm{CH}_{3}\right)$. IR $\left(\mathrm{KBr}, v_{\max }, \mathrm{cm}^{-1}\right): 2930 \mathrm{C}-\mathrm{H}$ of $\left.\mathrm{CH}_{3}\right), 1174(\mathrm{C}-\mathrm{S})$, $1574(\mathrm{C}=\mathrm{N}), 1176(\mathrm{C}-\mathrm{OH}), 1446(\mathrm{C}=\mathrm{C}), 1395(\mathrm{C}=\mathrm{C}), 1133$ $(\mathrm{C}=\mathrm{C}), 717(\mathrm{C}-\mathrm{S}), 525(\mathrm{~S}-\mathrm{S})$. CHNS analysis calcd. (found) $\%$ : C, 34.16(34.22). H, 2.51 (2.56), N, 34.85 (34.91) S, 22.80 (22.91). HRMS: $m / z\left(\mathrm{EI}^{+}\right)$calcd. for $\mathrm{C}_{8} \mathrm{H}_{7} \mathrm{~N}_{7} \mathrm{OS}_{2} 281.0153\left(\mathrm{M}+\mathrm{H}^{+}\right)$, found 282.0157 $\left(\mathrm{M}+\mathrm{H}^{+}\right)$.

2-((7H-Purin-6-yl)disulfanyl)-5-methyl-1,3,4-thiadiazole (compound 9): Yellow-orange solid, Yield: $76 \%$, m.p. $275^{\circ} \mathrm{C} .{ }^{1} \mathrm{H}$ NMR (DMSO- $\left.d_{6}\right): \delta 14.78$ (s, 1H, NH 6-MP), 8.87 (s, 1H, CH=N 6-MP), 8.12 (s, 1H, CH=N), 2.68 (s, 3H, $\left.\mathrm{CH}_{3}\right)$. IR $\left(\mathrm{KBr}, v_{\max }, \mathrm{cm}^{-1}\right): 1177(\mathrm{C}-\mathrm{S}), 1598(\mathrm{C}=\mathrm{N}), 1456(\mathrm{C}=\mathrm{C}), 1388$ $(\mathrm{C}=\mathrm{C}), 1137(\mathrm{C}=\mathrm{C}), 735(\mathrm{C}-\mathrm{S}), 566(\mathrm{~S}-\mathrm{S})$. CHNS analysis calcd. (found) \%: C, 34.03 (34.11), H, 2.14 (2.27), N, 29.76 (29.68), S, 34.07 (34.19). HRMS: $m / z\left(\mathrm{EI}^{+}\right)$calcd. for $\mathrm{C}_{8} \mathrm{H}_{6} \mathrm{~N}_{6} \mathrm{~S}_{3} 281.9816$ $\left(\mathrm{M}+\mathrm{H}^{+}\right)$, found $282.9921\left(\mathrm{M}+\mathrm{H}^{+}\right)$.

6-((1H-1,2,4-Triazol-3-yl)disulfanyl)-7H-purine (compound 10): Yellowish powder, , Yield: $80 \%$, m.p. $195{ }^{\circ} \mathrm{C} .{ }^{1} \mathrm{H}$ NMR (DMSO- $\left.d_{6}\right): \delta 16.95$ (s, 1H, NH triazole), 15.46 (s, 1H, NH 6-MP), 9.00 (s, 1H, CH=N 6-MP), 8.78 (s, 1H, $\mathrm{CH}=\mathrm{N}$ triazole), 8.12 (s, 1H, CH=N 6-MP). IR(KBr, $\left.v_{\max }, \mathrm{cm}^{-1}\right): 1171(\mathrm{C}-\mathrm{S}), 1588$ $(\mathrm{C}=\mathrm{N}), 1459(\mathrm{C}=\mathrm{C}), 1383(\mathrm{C}=\mathrm{C}), 1134(\mathrm{C}=\mathrm{C}), 737(\mathrm{C}-\mathrm{S}), 568$ (S-S). CHNS analysis calcd. (found) \%: C, 33.46 (33.51), H, 2.01 (2.07), N, 39.02 (39.18), S, 25.52 (25.59). HRMS: $m / z\left(\mathrm{EI}^{+}\right)$ calcd. for $\mathrm{C}_{7} \mathrm{H}_{5} \mathrm{~N}_{7} \mathrm{~S}_{2} 251.0048\left(\mathrm{M}+\mathrm{H}^{+}\right)$, found $252.0324\left(\mathrm{M}+\mathrm{H}^{+}\right)$. 
6-((5-(Furan-2-yl)-4H-1, 2,4-triazol-3-yl)disulfanyl)-7Hpurine (compound 11): Dark yellow powder, Yield: $82 \%$, m.p. $215^{\circ} \mathrm{C},{ }^{1} \mathrm{H}$ NMR (DMSO- $d_{6}$ ): $\delta 14.37$ (s, $1 \mathrm{H}, \mathrm{NH} 6-\mathrm{MP}$ ), 8.99 (s, 1H, CH=N 6-MP), 8.18-8.10 (m, 2H, CH=N 6-MP, $=\mathrm{CH}-\mathrm{O}$ furan $), 7.10(\mathrm{~d}, J=7.9 \mathrm{~Hz}, 1 \mathrm{H}, \mathrm{CH}=\mathrm{C}$ furan $), 6.71(\mathrm{t}$, $J=7.5 \mathrm{~Hz}, 1 \mathrm{H}, \mathrm{CH}=\mathrm{CH}$ furan $)$. IR $\left(\mathrm{KBr}, v_{\max }, \mathrm{cm}^{-1}\right): 1181(\mathrm{C}-\mathrm{S})$, 1558(C=N), $1449(\mathrm{C}=\mathrm{C}), 1393(\mathrm{C}=\mathrm{C}), 1144(\mathrm{C}=\mathrm{C}), 747(\mathrm{C}-\mathrm{S})$, 558 (S-S). CHNS analysis calcd. (found) \%: C, 41.63 (41.51), H, 2.22 (2.27), N, 30.90 (30.98), S, 20.21 (20.59). HRMS: $\mathrm{m} / 2$ $\left(\mathrm{EI}^{+}\right)$calcd. for $\mathrm{C}_{11} \mathrm{H}_{7} \mathrm{~N}_{7} \mathrm{OS}_{2} 317.0153\left(\mathrm{M}+\mathrm{H}^{+}\right)$, found 318.0025 $\left(\mathrm{M}+\mathrm{H}^{+}\right)$.

2-((7H-Purin-6-yl)disulfanyl)-5-phenyl-1,3,4-oxadiazole (compound 12): Yellowish powder, Yield: $68 \%$, m.p. $179^{\circ} \mathrm{C},{ }^{1} \mathrm{H}$ NMR (DMSO- $d_{6}$ ): $\delta 14.79$ (s, $1 \mathrm{H}, \mathrm{NH}$ 6-MP), 8.85 (s, 1H, CH=N 6-MP), 8.12 (s, 1H, CH=N 6-MP), 7.98 (d, $J=$ $6.8 \mathrm{~Hz}, 2 \mathrm{H}, \mathrm{CH}$ arom.), 7.61 (dt, $J=14.9,7.1 \mathrm{~Hz}, 3 \mathrm{H}, \mathrm{CH}$ arom.). $\mathrm{IR}\left(\mathrm{KBr}, v_{\max }, \mathrm{cm}^{-1}\right): 1181(\mathrm{C}-\mathrm{S}), 1558(\mathrm{C}=\mathrm{N}), 1449(\mathrm{C}=\mathrm{C})$, $1393(\mathrm{C}=\mathrm{C}), 1144(\mathrm{C}=\mathrm{C}), 747(\mathrm{C}-\mathrm{S}), 558(\mathrm{~S}-\mathrm{S})$. CHNS analysis calcd. (found) \%: C, 47.55 (47.53), H, 2.46 (2.27), N, 25.59 (25.68), S, 19.59 (19.59). HRMS: $m / z\left(\mathrm{EI}^{+}\right)$calcd. for $\mathrm{C}_{13} \mathrm{H}_{8} \mathrm{~N}_{6} \mathrm{OS}_{2} 328.0201$ $\left(\mathrm{M}+\mathrm{H}^{+}\right)$, found $329.9725\left(\mathrm{M}+\mathrm{H}^{+}\right)$.

Antitumor activity: The cells were planted under regular circumstances at $37^{\circ} \mathrm{C}$ in a $5 \% \mathrm{CO}_{2}$ moistened environment, either in Dulbecco's adjusted eagle medium or adjusted eagle medium, (contingent on cell line), supplemented with $10 \%$ fetal calf plasma (Biosera, U.K.), $1 \%$ L-glutamine, $1 \%$ non-vital amino acids and $0.05 \%$ hydrocortisone (Gibco, Invitrogen, USA). Cells were scattered in 96-well plates in a whole quantity of $160 \mathrm{~mL}$ and were permitted to attain a 30-40\% degree of convergence before beginning the test. The compounds were suspended in distilled ultrapure water at upper limit strength of $100 \mathrm{mM}$ and consecutive decimal concentrations were made. These were supplemented with the cells in a size of $40 \mathrm{~mL}$, following $96 \mathrm{~h}$ of constant exposure to the agent; the cytotoxicity was verified by MTT [3-(4,5-dimethylthiazol-2-yl)2,5-diphenyltetrazolium bromide] (Kings Synthesis Ltd. UK) colorimetric method. Absorbance was measured by spectrophotometry at $\lambda=570 \mathrm{~nm}$ (ELx808, Bio-Tek Instruments, Inc.). $\mathrm{IC}_{50}$ values were measured by a dose-response evaluation applying Origin $6.0^{\circledast}$ software that provides directly the $\mathrm{IC}_{50}$. Assessment of cytotoxicity of potential clinical candidates utilizing different cancer cell lines established from clinical tumor samples can offer a preliminary insight into the mechanism of action and potential secondary effects.

\section{RESULTS AND DISCUSSION}

Cytotoxicity screening: Tests to evaluate the capability of compounds to supply temporary cell growth inhibition as well as long-term growth inhibition study with preliminary result of short term. The first method that implemented these needs used a tetrazolium salt to detect and quantify metabolically active cells. In vitro antitumor action of compounds $\mathbf{5}$ and 6 against amurine leukemia cell line (L1210) (Fig. 2), a human leukocyte cellline (HL60) (Fig. 3) and chronic lymphocytic leukemia cells were estimated utilizing the MTT test. The effects confirm that compounds $\mathbf{3 , 5}, \mathbf{8}$ and to lesser extent compound $\mathbf{2}$ is significantly more cytotoxic than rest of compounds for the tested cells $\left(\mathrm{IC}_{50}\right.$ value range $(6.2-12.2 \mathrm{M})$ than

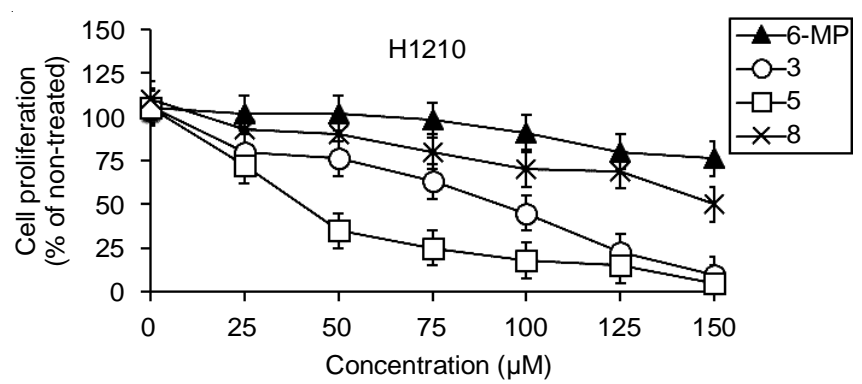

Fig. 2. Effects of 6-MP, 3, $\mathbf{5}$ and $\mathbf{8}$ compounds on HL1210 cell proliferation utilizing the MTT test. The data appeared are the mean \pm SEM

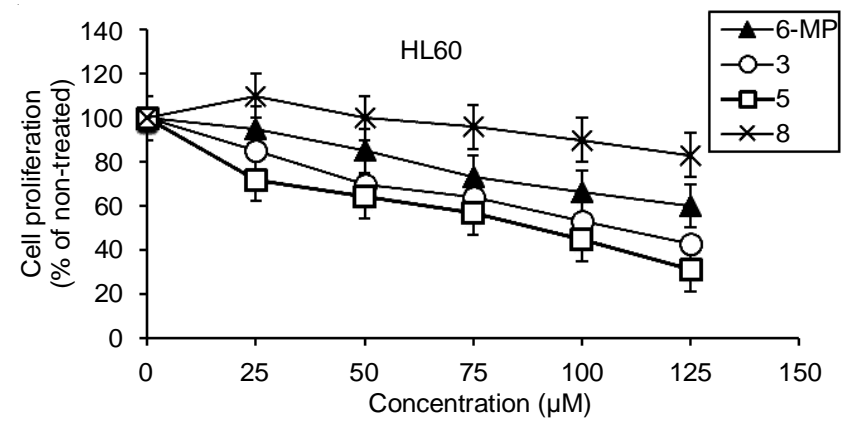

Fig. 3. Effects of 6-MP, $\mathbf{3}, \mathbf{5}$ and $\mathbf{8}$ compounds on HL60 cell proliferation utilizing the MTT test. The data appeared are the mean \pm SEM

6-MP value (9.3-12.6 mM) (Table-1). More interestingly, compounds having free $\mathrm{OH}$ phenolic group display more intense action than the other tried compounds. These fundamental outcomes recommend that substitution of C-3 phenyl group by pyridine decrease cytotoxic effect two functionalized pharmacophores (for this situation triazole and free - $\mathrm{OH}$ group) are ideal for activity. Substitution of -NH triazole with sulfur or oxygen as in compounds $\mathbf{9 ,} \mathbf{1 1}$ and $\mathbf{1 2}$ decrease activity while the addition of methyl or $\mathrm{NH}_{2}$ group to triazole significantly enhance activity as seen in compounds $\mathbf{3}$ and $\mathbf{8}$, the results could encourage further modificationsfor optimization of results. In vitro handling of CLL-119 cells by compounds 3,5 and 8 at $50 \mathrm{M}$ for $48 \mathrm{~h}$ revealed a notable improvement in the amount of apoptotic (Fig. 4) and non-living cells (Fig. 5), correlated with 6-mercaptopurine. The initial \% of non-living cells in a non-handled cell group was about $45 \%$. Non-living cell

TABLE-1

SHORT-TERM CANCER INHIBITORY ACTIVITIES OF THE DEVELOPED COMPOUNDS (1-12) TOWARD L1210, HL60 AND CCL-119 CELLS IN THE MTT TEST $\left(\mathrm{IC}_{50}, \mu \mathrm{M}\right)$

\begin{tabular}{crrr}
\hline \multirow{2}{*}{ Compd. } & \multicolumn{3}{c}{$\mathrm{IC}_{50}(\mu \mathrm{M})$} \\
\cline { 2 - 4 } & \multicolumn{1}{c}{$\mathrm{L} 1210$} & \multicolumn{1}{c}{ HL60 } & \multicolumn{1}{c}{ CCL-119 } \\
\hline 1 & $16.3 \pm 0.20$ & $24.7 \pm 0.5$ & $19.2 \pm 0.3$ \\
2 & $21.5 \pm 0.10$ & $7.8 \pm 0.6$ & $10.8 \pm 0.4$ \\
3 & $11.9 \pm 0.20$ & $6.2 \pm 0.2$ & $8.3 \pm 0.1$ \\
4 & $24.5 \pm 0.50$ & $9.4 \pm 0.1$ & $15.6 \pm 0.2$ \\
5 & $9.8 \pm 0.70$ & $12.2 \pm 1.3$ & $8.6 \pm 0.4$ \\
6 & $16.8 \pm 0.01$ & $15.6 \pm 0.2$ & $13.3 \pm 0.1$ \\
7 & $21.2 \pm 0.20$ & $37.3 \pm 0.4$ & $16.7 \pm 0.6$ \\
8 & $7.4 \pm 0.40$ & $10.4 \pm 0.1$ & $5.3 \pm 0.4$ \\
9 & $16.2 \pm 0.10$ & $37.2 \pm 0.3$ & $36.2 \pm 0.8$ \\
10 & $14.8 \pm 0.30$ & $22.2 \pm 0.8$ & $14.9 \pm 0.3$ \\
11 & $11.3 \pm 0.30$ & $11.8 \pm 1.1$ & $24.3 \pm 0.6$ \\
12 & $18.2 \pm 0.70$ & $10.2 \pm 1.2$ & $14.3 \pm 0.3$ \\
$6-\mathrm{MP}$ & $9.7 \pm 1.20$ & $11.3 \pm 0.7$ & $12.4 \pm 0.2$ \\
\hline
\end{tabular}




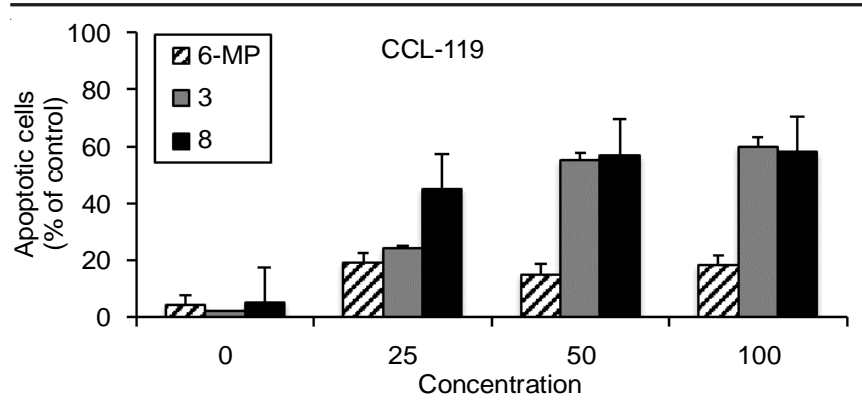

Fig. 4. Apoptotic CLL-119 cells exposed to 6-MP compound $\mathbf{3}$ and $\mathbf{8}$

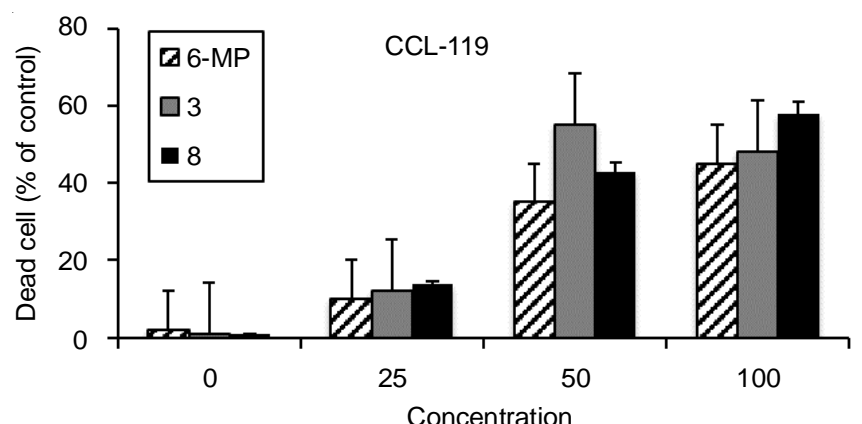

Fig. 5. Viability of cells with compounds $\mathbf{3}, \mathbf{8}$ and 6-MP, between 0 and $100 \mu \mathrm{M}$, for $16 \mathrm{~h}$ at $37^{\circ} \mathrm{C}$

number following therapy with compound $\mathbf{3}$ or $\mathbf{8}$ were 76 and about $54 \%$ each $(\mathrm{p}<0.001)$, while therapy with 6 -mercaptopurine, provided a moderate influence, about $48 \%(\mathrm{p}<0.01)$, which was not an essential variation as correlated to non-handled cell.

Herein, twelve 6-mercaptopurine analogs bearing 1,2,4substituted triazoles linked as disulfide prodrugs. These have certain advantages beyond those in the modern therapeutic applications. Among those benefits are a higher overall cytotoxic action, greater cell layer penetration and few detrimental side influences, owing essentially to the small dosages used [23]. The enhanced features of the new derivative attributed in part to couple different biological fragments in the same molecule. The substituted 1,2,4-triazole conjugate SH functionality at the exterior of cell while 6-mercaptopurine drug serves as purine antimetabolite drug utilizing DNA damaging effect. Great cellwall penetration is owing to lipophilic characteristics of the aromatic ring or alkyl substituent on triazole ring that comprises three nitrogen molecules and be able to serve as a hydrogen bond donor or acceptor at the functioning position of receptors and have capacity to change their activities subsequently. The triazole moiety can improve the solubility of drug as a whole due to polarity nature and provide better pharmacokinetic and pharmacodynamics features.

\section{Conclusion}

Disulfide prodrugs of 6-mercaptopurine were effectively synthesized and characterized through spectroscopic methods as well as elemental analysis. Antitumor activities were evaluated by MTT method, the results showed that they have effective actions related to 6-mercaptopurine. Compounds $\mathbf{3}, \mathbf{5}$ and $\mathbf{8}$ were found to have good activities with levels of $\left(\mathrm{IC}_{50} \mu \mathrm{M}\right.$ : $5.8 \pm$ 0.1 and $8.6 \pm 0.5$ respectively against CCL- 119 which is relatively better than 6-mercaptopurine for the same experiment (12 \pm 0.4 ). Compounds $\mathbf{2}, \mathbf{3}$ and $\mathbf{4}$ seems to have fair activity against $\mathrm{HL} 60$ of $\left(\mathrm{IC}_{50} \mu \mathrm{M}: 6.2 \pm 0.2\right.$ and $9.7 \pm 0.4$ while compound
3 give better activity against CCL-119 and these results were confirmed by MTT method.

\section{CONFLICT OF INTEREST}

The authors declare that there is no conflict of interests regarding the publication of this article.

\section{REFERENCES}

1. K. Schmiegelow, S.N. Nielsen, T.L. Frandsen and J. Nersting, J. Pediatr. Hematol. Oncol., 36, 503 (2014); https://doi.org/10.1097/MPH.0000000000000206.

2. B.A. Kamen, J. Pediatr. Hematol. Oncol., 31, 383 (2009); https://doi.org/10.1097/MPH.0b013e3181a6e191.

3. S.A. Coulthard, L.A. Hogarth, M. Little, E.C. Matheson, C.P.F. Redfern, L. Minto and A.G. Hall, Mol. Pharmacol., 62, 102 (2002); https://doi.org/10.1124/mol.62.1.102.

4. N.V. Bhagavan and C.-E. Ha, Essentials of Medical Biochemistry, edn 2, pp. 465-487 (2015).

5. J.H. Block and J.M. Beale, Wilson and Gisvold's Textbook of Organic Medicinal and Pharmaceutical Chemistry, Lippincott Williams \& Wilkins, edn 12, pp. 378-379 (2011).

6. O.H. Nielsen, B. Vainer and J. Rask-Madsen, Aliment. Pharmacol. Ther, 15, 1699 (2001);

https://doi.org/10.1046/j.1365-2036.2001.01102.x.

7. I. Tiede, G. Fritz, S. Strand, D. Poppe, R. Dvorsky, D. Strand, H.A. Lehr, S. Wirtz, C. Becker, R. Atreya, J. Mudter, K. Hildner, B. Bartsch, M. Holtmann, R. Blumberg, H. Walczak, H. Iven, P.R. Galle, M.R. Ahmadian and M.F. Neurath, J. Clin. Invest., 111, 1133 (2003); https://doi.org/10.1172/JCI16432.

8. C. Cuffari, S. Hunt and T.M. Bayless, Aliment. Pharmacol. Ther, 14, 1009 (2000);

https://doi.org/10.1046/j.1365-2036.2000.00812.x

9. E. Raetz and J. Meyer, Hematologist, 13, 1 (2016).

10. K.K. Das, H.T. Nishino and A.T. Chan, Inflamm. Bowel Dis., 16, 1454 (2010); https://doi.org/10.1002/ibd.21205.

11. G.H. Elgemeie, Curr. Pharm. Des., 31, 2627 (2003); https://doi.org/10.2174/1381612033453677.

12. M. Arenas, G. Simpson and C.M. Lewis, Clin. Chem., 51, 2371 (2005); https://doi.org/10.1373/clinchem.2005.053157.

13. N.K. De Boer, A.A. van Bodegraven, B. Jharap, P. de Graaf and C.J. Mulder, Nat. Clin. Pract. Gastroenterol. Hepatol., 4, 686 (2007); https://doi.org/10.1038/ncpgasthep 1000.

14. L.J. Derijks and D.R. Wong, Curr. Pharm. Des., 16, 145 (2010); https://doi.org/10.2174/138161210790112773.

15. K.B. Sloan, S.C. Wasdo and J. Rautio, Pharm. Res., 23, 2729 (2006); https://doi.org/10.1007/s11095-006-9108-0.

16. K.B. Sloan and S.C. Wasdo, ed.: M. Roberts and K. Walters, The Role of Prodrugs in Penetration Enhancement, In: Dermal Absorption and Toxicity Assessment, Marcel Dekker: New York, pp. 605-622 (2008).

17. D.R. Wong, L.J. Derijks, M.O. den Dulk, E.H. Gemmeke and P.M. Hooymans, Ther. Drug Monit., 29, 845 (2007); https://doi.org/10.1097/FTD.0b013e31815bf4dc.

18. R. Kaur, A.R. Dwivedi, B. Kumar and V. Kumar, Anticancer. Agents Med. Chem., 16, 465 (2016); https://doi.org/10.2174/1871520615666150819121106.

19. M. Galanski, V. Arion, M. Jakupec and B. Keppler, Curr. Pharm. Des., 9, 2078 (2003); https://doi.org/10.2174/1381612033454180.

20. Z. Li, Z. Gu, K. Yin, R. Zhang, Q. Deng and J. Xiang, Eur. J. Med. Chem., 44, 4716 (2009); https://doi.org/10.1016/j.ejmech.2009.05.030.

21. J. Lee, S.J. Kim, H. Choi, Y.H. Kim, I.T. Lim, H.M. Yang, C.S. Lee, H.R. Kang, S.K. Ahn, S.K. Moon, D.-H. Kim, S. Lee, N.S. Choi and K.J. Lee, J. Med. Chem., 53, 6337 (2010); https://doi.org/10.1021/jm1002414.

22. Y.B. Zhang, W. Liu, Y.S. Yang, X.L. Wang, H.L.Zhu, L.F. Bai and X.Y. Qiu, Med. Chem. Res., 22, 1 (2013).

23. K. Nepali, S. Sharma, R. Ojha and K.L. Dhar, Med. Chem. Res., 22, 1 (2013); https://doi.org/10.1007/s00044-012-0002-5. 